\title{
¿QUÉ ES UNA LEY IRREDUCTIBLEMENTE ESTADÍSTICA? EL CASO DE LA MECÁNICA CUÁNTICA
}

SERGIO MARTINEZ

INSTTIUTO DE INVESTTGACIONES FILOSÓFICAS

UNAM

\section{Introducción}

En una serie de trabajos el profesor Ian Hacking ha esbozado la historia del desarrollo de la noción de "autonomía de leyes estadísticas" en la ciencia (véase Hacking, 1983a y 1983b, 1990). Según Hacking este desarrollo culmina con la prueba de la no existencia de variables ocultas por Von Neumann en 1932, cuando se introduce explícitamente en la ciencia el concepto de "ley estadística irreductiblemente autónoma”. Una ley estadística es una ley que se expresa en términos de una asignación de probabilidades a eventos o propiedades. Una ley estadística irreductiblemente autónoma es aquella que involucra probabilidades (que se consideran) irreducibles a leyes deterministas. Quiero mostrar en este trabajo algunas de las dificultades que afronta la clarificación de la idea de irreductibilidad estadística a través de un examen del ejemplo paradigmático de tal tipo de ley en la mecánica cuántica.

El concepto de ley de la naturaleza estadística (o probabilista) irreductiblemente autónoma está en el fondo de una serie de cuestiones filosóficas planteadas por el uso de métodos estadísticos en la ciencia, así como en discusiones sobre temas tan fundamentales como lo son la interpretación de las probabilidades y la estructura de la causalidad física. Estos temas sin embargo, no seran tratados aquí. En este trabajo nos interesa la presentación y la discusión de un aspecto del problema filosófico de fondo. ${ }^{1}$ Muchas discusiones sobre estos temas en la filosofia de la ciencia toman a la ligera las dificultades que presenta la formulación y la caracterización de una ley de la naturaleza irreductiblemente estadística.

La mecánica cuántica se caracteriza por la presencia de un algoritmo que podemos llamar algoritmo cuántico, según el cual los enunciados básicos de la teoría son de la forma "la probabilidad de que una magnitud (observable) $M$

1 En Martínez (1989) examino un aspecto más técnico del mismo problema, a saber, el problema de la interpretación del postulado de la proyección en la mecánica cuántica. 
tenga un valor incluido en un intervalo $I$ de números reales (y más en general, en un conjunto de Borel), para un sistema en el estado inicial $p$ es $r$. Donde $r$ toma valores en el intervalo $[0,1]$ ". Para visualizar esta idea pensemos en un sistema cuántico tal que una de sus magnitudes puede tomar sólo dos valores, " 0 " y " 1 ", por ejemplo. Supongamos que el algoritmo cuántico asocia a ambos valores probabilidad 0.5 . El resultado de cada medición (observación) es tan azaroso como el resultado de lanzar una moneda al aire. ${ }^{2}$ Pero conforme el número de mediciones $n$ (de la misma magnitud en el mismo estado) aumenta, la ley estadística se prefigura cada vez más claramente. El número de ceros y unos tiende a igualarse, con mayor probabilidad cuanto mayor es $\boldsymbol{n}$. En este sentido la ley estadística "surge del azar".

Ya con las formulaciones iniciales de la teoría se pensó que las probabilidades del algoritmo cuántico eran irreducibles. Pero scómo podemos excluir la mera posibilidad de la existencia de una teoría más general determinista que permita una reducción (por variables ocultas) de las probabilidades generadas por el algoritmo cuántico? Esta pregunta está íntimamente relacionada con otra, ¿qué significa, a nivel de leyes de la naturaleza, las probabilidades del algoritmo cuántico?

Hay dos estrategias posibles a través de las cuales podemos enfocar el problema. Una estrategia es tratar de interpretar la irreductibilidad a través de una interpretación de la mecánica cuántica. Como veremos, el problema es que no hay un consenso respecto a qué puede considerarse como una interpretación satisfactoria, y lo que es más importante para nuestro problema, diferentes interpretaciones (que satisfacen ciertos requisitos mínimos de adecuación empírica) llevan a interpretaciones radicalmente diferentes de las probabilidades generadas por el algoritmo cuántico. La otra estrategia es tratar de establecer, independientemente de los aspectos controversiales de la interpretación de la teoría, la imposibilidad de construir una teoría determinista más general que satisfaga ciertos requisitos mínimos de adecuación empírica. Esto nos lleva al tema de las teorías de variables ocultas en la mecánica cuántica.

Después de examinar estas dos estrategias mostraremos en qué sentido la irreductibilidad puede establecerse sobre la base del teorema de Bell. Aunque, como haremos ver, el significado de tal irreductibilidad es todavía bastante oscuro.

2 Aquí dejamos de lado un problema serio, hasta qué punto es el azar cuántico el mismo que el azar clásico. La respuesta depende de cómo definamos el azar y sobre todo de cómo distingamos entre azar matemático y azar físico. Asumimos aquí que podemos definir un concepto de azar físico que abarque los ejemplos arriba mencionados. Véase por ejemplo la discusión en el capítulo 8 de Earman (1986). 


\section{El problema de la interpretación y sus implicaciones para la noción de irreductibilidad}

Hay dos aspectos del problema filosófico de interpretación de una teoría física y de la mecánica cuántica en particular que imponen condiciones para una interpretación satisfactoria. Por un lado, se requiere una interpretación física del formalismo matemático. Tal y como lo entendemos aquí, esto consiste en generar una serie de modelos de diferentes grados de abstracción que nos convenzan de la adecuación empírica de la teoría. En último término podemos aspirar a un modelo semántico que funcione como un requerimiento de consistencia. En muchos casos podemos resolver satisfactoriamente el problema filosófico de interpretación por medio de una interpretación por modelos de un sistema axiomático explícito o implícito. En el caso de la mecánica cuántica, sin embargo, no puede hacerse una distinción clara entre cuestiones semánticas y cuestiones metafísicas, y esto nos lleva a tener que considerar explícitamente requerimientos no puramente semánticos de adecuación. ${ }^{3}$

Estos requerimientos no semánticos pueden ser de diversos tipos. Por ejemplo, la interpretación de Bohr de la que hablaremos brevemente a continuación considera relevantes aspectos puramente epistemológicos de adecuación. Bub, por el contrario, requiere un cambio a nivel de la estructura lógica de los eventos en el mundo (véase Bub, 1974, 1977).

Esto presupone que la lógica clásica no describe la estructura lógica de los eventos a nivel microscópico. A su vez, la interpretación por mundos paralelos por el contrario no cuestiona la estructura lógica fundamental de los eventos, sino más bien cuestiona la ontología básica del mundo. Luego evaluaremos aspectos de estas propuestas relacionadas con nuestro problema. Por ahora sólo se introducen para ejemplificar la distinción entre estos dos tipos de requerimientos. Una vez que no podemos delimitar claramente el aspecto semántico de una teoría de cuestiones metodológicas y/o metafísicas, el problema de interpretación de una teoría se torna sumamente complejo.

El contraste entre las ideas de Bohr y Heisenberg es un buen ejemplo del uso al que queremos poner la distinción entre requerimientos interpretativos que introdujimos anteriormente.

Bohr siempre insistió en que el problema de interpretación no era un problema (semántico) de interpretación de una teoría formal, y que no era suficiente clarificar la aplicabilidad de la teoría a situaciones concretas a partir de la interpretación de un formalismo matemático. ${ }^{4}$ Heisenberg, por el con-

$3 \mathrm{Mi}$ interés aquí no es tratar de caracterizar estos requerimientos de una manera exhaustiva y abstracta, sino hacer ver que existen y que juegan un papel muy importante en el problema de interpretación de la mecánica cuántica y por ende en el problema de interpretar el concepto de irreductibilidad estadística.

4 Para una exposición de la filosofía de la naturaleza de $\mathrm{N}$. Bohr véanse por ejemplo Mackinnon (1982) y Folse (1985). 
trario, tendía a ver el problema como un mero problema de interpretación del formalismo. Cuando Heisenberg probó el teorema que establece las relaciones de incertidumbre, de acuerdo con las cuales es imposible definir el estado del sistema físico con valores precisos de las magnitudes conjugadas canónicas, Bohr rechazó la pretensión de Heisenberg de que esto nos permitía finalmente resolver el problema de interpretación. Para Bohr la solución del problema de interpretación requería algo más que la interpretración empírica de una relación entre operadores, requería llegar a entender la relación eritre el formalismo y el uso de los conceptos clásicos necesarios para describir sistemas físicos microscópicos. Heisenberg por el contrario, parecía jugar libremente con interpretaciones posibles sin sujetarse a ningún canon metodológico interpretativo más allá de los requerimientos impuestos por una interpretación del formalismo mínimamente adecuada. Esto llevó a Heisenberg a sugerir muchas ideas contradictorias. Después de tratar de justificar su interpretación (de las relaciones de incertidumbre), sobre la base de una teoría verificacionista del significado, Heisenberg desarrolla luego una ontología de "potencias" según la cual un sistema cuántico tiene propiedades potencialmente que sólo se actualizan a través de mediciones. Esta ontología, bastante común en libros de texto y popularizaciones, tal y como fue sugerida por Heisenberg, resulta ser inconsistente con las leyes dinámicas de la teoría (véanse Wigner, 1963; Shimony, 1974).

La así llamada interpretación de Copenhague es una mezcla confusa e informe de las ideas de Bohr y Heisenberg. La mayoría de las versiones de esta interpretación no reconocen ni siquiera implícitamente el hecho de que Bohr y Heisenberg partían de presupuestos metodológicos distintos. Aquí nos concentramos en la interpretación que puede atribuirse a Bohr y que en lo que nos atañe forma parte esencial de las diferentes versiones de la así llamada interpretación de Copenhague.

\section{La interpretacion de Bobr}

De acuerdo con Bohr, el estado clásico del sistema aislado que debe definirse para predecir el comportamiento (estado) futuro del sistema, no está literalmente determinado por "observación". Ya que “observación”, literalmente, involucra una interacción con el sistema que no nos permite considerar el sistema objeto de manera aislada, lo que es una suposición necesaria de la física clásica para definir el estado del sistema. En el límite de mediciones que requieren poca energía con respecto a la magnitud medida, como por ejemplo cuando se mide la velocidad de un automóvil o un planeta, podemos despreciar la perturbación causada por la medición en el sistema aislado y aproximar 
por medio de la medición el valor de los parámetros que caracterizan el estado. 5

El eje de la interpretación de Bohr es el papel epistemológicamente privilegiado que le asigna a la física clásica. Esto le permite interpretar las probabilidades del algoritmo como si reflejara, por así decir, la distancia entre nuestro aparato conceptual clásico, un producto refinado de nuestra experiencia cotidiana, y la estructura del mundo a un nivel que no es directamente accesible a nuestra experiencia. Bohr entonces interpreta las probabilidades del algoritmo cuántico como la expresión cuantitativa de la limitada aplicación del marco conceptual clásico al dominio atómico.

Entender el origen de la irreductibilidad del algoritmo cuántico de esta manera es, sin embargo, deficiente en un sentido crucial. Esta interpretación epistemológica de la irreductibilidad del algoritmo cuántico asume que, como en el caso del lanzamiento de una moneda, existe una diferencia cualitativa clara entre los dos niveles físicos que podemos relacionar por medio de una reducción por variables ocultas. ${ }^{6}$

En el caso del lanzamiento de una moneda (visto como un experimento con resultado cara o cruz) las magnitudes macroscópicas observadas se interpretan como magnitudes representativas de valores de magnitudes microscópicas que se "pierden" en el proceso del lanzamiento de la moneda debido a la inestabilidad del proceso con respecto a condiciones iniciales (mecánicas) microscópicas. ${ }^{7}$ En este caso interpretamos el azar por medio de una relación de variables ocultas a una teoría determinista. La intuición básica detrás de la interpretación de Bohr de las probabilidades cuánticas parece ser la siguiente. En el caso del lanzamiento de una moneda podemos reconstruir, por lo menos en principio, a partir de nuestra experiencia (observaciones) con procesos macroscópicos, el proceso mecánico que explica el azar aparente que observamos (a nivel macroscópico). Si generalizamos a partir de este ejemplo podemos pensar que un proceso azaroso puede detectarse epistemológicamente a nivel de magnitudes macroscópicas (y en general a nivel de las magnitudes a reducirse) como limitaciones en la exactitud de observaciones. Y que ésta es la única forma en que podemos generar una teoría de variables ocultas para explicar (reducir) el azar. Pero esto no es así.

Presento a continuación un ejemplo en el que el azar tiene un origen diferente al del ejemplo del lanzamiento de la moneda. Este es un tipo de azar

5 Esta imposibilidad literal de determinar la posición en un tiempo dado simultáneamente con la determinación del sistema en la mecánica clásica da lugar, junto con la hipótesis empírica del postulado cuantico, a la famosa doctrina de la complementariedad de Bohr.

6 Véase la sección 5 para una clarificación de esta de reducibilidad por variables ocultas. Véase la nota 11 en particular.

7 Esta es una relación por variables ocultas que siempre se ha asumido es posible construir, pero que sólo recientemente se ha construido rigurosamente. Véase Keller (1986) por ejemplo. 
que no proviene de la inexactitud del registro de las condiciones iniciales y que por lo tanto sugiere un origen distinto del azar del que Bohr asumió en su análisis. A su vez esto sugiere la posibilidad de una reducción por variables ocultas que no estaría excluido por el análisis epistemológico de Bohr sobre la que él asienta su interpretación.

El ejemplo de las dos estrellas. ${ }^{8}$ La siguiente situación es físicamente posible de acuerdo con la mecánica newtoniana. Dos estrellas con la misma masa giran alrededor de su centro de gravedad. Se sigue de las leyes de Newton que las dos estrellas viajan con la misma velocidad en una órbita circular. El plano que contiene a la órbita circular lo podemos llamar $P$; al eje de simetría, que pasa por el centro del círculo y es perpendicular a $P$, lo llamamos $E$. Un cometa, que llamaremos el cometa $C$, se mueve a lo largo de $E$. Cerca de una de las estrellas hay un planeta llamado $W$, habitado por astrónomos que han mantenido records muy precisos por milenios de las apariciones del cometa cuando el cometa cruza el plano $P$, que es cuando el cometa está más cerca del planeta.

En este mundo se considera al cometa como un mensajero del diablo, porque a pesar del esfuerzo de todos los astrónomos del planeta $W$, nunca pueden predecir su próxima aparición. Es más, records de miles de años muestran que los astrónomos han tenido el mismo nivel de éxito en las predicciones que los astrólogos observando su bola de cristal. Los astrónomos del planeta $W$ tal vez nunca llegarán a saberlo, pero como Sitnikov (1960) ha demostrado, no existe un algoritmo que permita predecir su próxima aparición. Aun cuando los astrónomos de $W$ tuvieran una información totalmente precisa de todas las veces que el cometa ha atravesado el plano $P$, ellos nunca podrían encontrar una regularidad legaliforme en las apariciones que les permitiría predecir la próxima aparición. Las leyes de la mecánica celeste son, en este caso, compatibles con cualquier sucesión futura de apariciones.

La próxima aparición del cometa es tan azarosa como el resultado de lanzar una moneda al aire. Existe, sin embargo, una diferencia crucial en las situaciones ejemplificadas en estos dos casos.

En el caso del lanzamiento de la moneda el azar se origina en la imprecisión de la información a nivel macroscópico. Es esta imprecisión en la información la que (según la hipótesis de la inestabilidad del sistema) nos permite interpretar las probabilidades como promedios de condiciones iniciales microscópicas. En el segundo ejemplo, sin embargo, la información a nivel macroscópico es tan precisa como queramos. El azar no surge de la falta de precisión en la información acerca de las condiciones iniciales. No hay ningún indicio epistemológico, dentro de la situación descrita, del origen de la información faltante que origina el azar.

8 Sigo aquí la versión de este ejemplo presentada por Ekeland (1984). 
Asumiendo un conocimiento de la mecánica celeste podemos entender fácilmente la situación. Los astrónomos del planeta $W$ no tienen conocimiento completo del estado dinámico clásico del sistema, no conocen la velocidad a la que el planeta atraviesa el plano $P$. Pero el hecho es que a menos que tengamos conocimiento de la mecánica clásica no existe en la situación descrita ningún indicio acerca de la dirección en que podemos encontrar el nivel reductor (consistente en magnitudes de una teoría más general) necesario para reducir el azar (aparente) a una teoría determinista, como en el caso del lanzamiento de la moneda.

El ejemplo de las dos estrellas nos proporciona un tipo de situación que podría "ocultarse” detrás del algoritmo cuántico que no es accesible a través del análisis epistemológico de Bohr. Si el algoritmo cuántico describiera un proceso con información precisa (completa) de cierto tipo de condiciones iniciales pero incompleto en sentido dinámico, como el ejemplo de las dos estrellas sugiere, sólo podríamos conceptualizar esa incompletitud desde otra teoría determinista que no estaría al alcance de un análisis de las limitaciones de la teoría al nivel de observaciones, tal y como Bohr parece sugerir. ${ }^{9}$ Parece ser que la única forma de reducir el azar en este caso sería desde la perspectiva de una teoria más general en la que el estado del sistema proporcione una descripción completa. Pero lo que no sabemos en el caso de la mecánica cuántica es precisamente si tal teoría existe. ${ }^{10}$

\section{Irreductibilidad en mundos paralelos}

Esta interpretación fue originalmente propuesta por Everett (véase DeWitt, 1973). Su idea básica es la siguiente. Tomemos el ejemplo introducido anteriormente sobre un sistema cuántico cuyas magnitudes pueden tomar sólo dos valores, "0" y " 1 ". Esta interpretación nos dice que en "en realidad" el sistema tiene ambos valores, sólo que en "mundos" diferentes. Además, para "salvar los fenómenos", esta interpretación requiere que estos mundos diferentes no tengan manera de saber de los otros mundos (y en este sentido son

9 Este argumento contra la interpretación de Bohr de la irreductibilidad de las probabilidades cuánticas lo hemos elaborado con más detalle, y desde una perspectiva ligeramente diferente en Martínez (1988).

10 Heisenberg la presenta con una variante de la idea de Bohr. El origen de la irreductibilidad cuántica, nos dice Heisenberg, es una perturbación ineliminable del objeto observado en el proceso de medición. Esta perturbación ineliminable se expresa en las famosas relaciones de incertidumbre de acuerdo con las cuales no es posible medir simultáneamente dos magnitudes complementarias (conjugadas) con exactitud arbitraria. El único argumento que tiene Heisenberg de que la perturbación es ineliminable, independiente del análisis del proceso de la observación de Bohr que hemos presentado anteriormente, es que las relaciones de incertidumbre pueden derivarse como un teorema matemático del formalismo de la teoría. Esto sin embargo no es suficiente. Después de todo, de haber variables ocultas, también podríamos derivar las relaciones de incertidumbre, aunque, por supuesto, no significarían lo mismo. 
“paralelos"), una vez que una interacción física (una medición por ejemplo) los hace diverger. Cada quien en su mundo piensa que su mundo es el único que existe.

Esta es una interpretación "realista" en el sentido que surge naturalmente del formalismo matemático y se refiere a entes teóricos que tienen propiedades en el sentido clásico, sólo que diferentes, en mundos (que podemos llamar) paralelos. Tiene la ventaja que el estado de un sistema físico no depende de una situación de medición y por lo tanto permite, en principio, la aplicación de la mecánica cuántica a la cosmología. No es un accidente que los principales proponentes de esta interpretación (como Geroch y Wheeler) sean cosmólogos.

Estos resultados le permitieron a Everett interpretar los resultados de mediciones, y por ende las probabilidades generadas por el algoritmo cuántico de forma consistente con su postulación del origen del problema, la diversidad de mundos. La intepretación es consistente, y sobre la base de la ontología postulada explica las aplicaciones de la teoría. Pero la mayoría de los intérpretes de la mecánica cuántica comparten con nosotros el rechazo a tal interpretación. Quizás, como decía Everett, se deba a que somos como los fanáticos creyentes en el mundo cerrado de Aristóteles que Copérnico destruyó. Sin adentrarnos en criticismos detallados, sin embargo, esta interpretación es sospechosa porque, parte de un supuesto difícilmente reconciliable con una epistemología científica mínimamente adecuada. ${ }^{11}$ :Cuáles son los criterios, o las condiciones físicas necesarias para generar una bifurcación de mundos? Supuestamente la medición lleva a una bifurcación de mundos, pero ¿cuántos de ellos, y cuándo se generan? Parece ser que en el fondo tendríamos que recurrir a la generación por medio de un acto mental de las bifurcaciones, o bien imponer por un acto de fe aquellas que nos sirven para explicar el algoritmo cuántico.

En esta interpretación la irreductibilidad simplemente desaparece en la metafísica (bajo supuestos epistemológicos ad hoc). En lugar de variables ocultas hay mundos ocultos inaccesibles a nuestra experiencia. En tanto que la metafísica de mundos posibles paralelos no pueda anclarse en una epistemología mínimamente adecuada no parece que pueda iluminarnos el problema de la interpretación de la irreductibilidad del algoritmo cuántico. Lo que sí hace esta interpretación es mostrarnos la necesidad de considerar requerimientos no semánticos en el análisis del problema.

11 Para una presentación clara y concisa de esta interpretación y de sus problemas, véase Rae (1986). Una presentación informal de los argumentos más importantes en favor y en contra de esta interpretación se encuentran en varias entrevistas publicadas en Davies y Brown (1986). Un examen detallado se encuentra en Healey (1984). 


\section{La interpretación de Bub}

Bub muestra cómo la teoría clásica de la probabilidad puede reconstruirse, de una forma muy natural, como un cálculo de operadores en el que el cálculo de operadores de la mecánica cuántica puede interpretarse como una teoría de la probabilidad generalizada. Esto permite formular el problema de la interpretación de la teoría cuántica y de la irreductibilidad en particular, como el problema de entender a nivel ontológico la transición entre dos estructuras algebraicas interpretadas como estructuras de posibilidad. Ésta es una formulación que ha clarificado importantes aspectos del problema pero que hasta ahora no ha llevado a una solución definitiva. Este enfoque del problema de interpretación presupone que la lógica de los eventos en el mundo microscópico no es la lógica clásica, sino la "lógica cuántica", una idea que encuentra una serie de dificultades desde los ángulos más diversos (véase por ejemplo Hellman, 1980). En el fondo la dificultad es la misma que enfren$\tan$ otras interpretaciones que se basan en una metafisica modal (como en el caso de las "potencias" de Heisenberg). A saber, la dificultad de interpretar las modalidades postuladas (implícitamente a través de la interpretación de la estructura algebraica como una estructura de posibilidad) en términos de experimentos que generan resultados definidos (actuales, no simplemente posibles).

Las interpretaciones que hemos presentado brevemente son casos extremos en la diversidad de interpretaciones que se han sugerido para la mecánica cuántica. En el contraste de estas interpretaciones podemos ver el problema de fondo para cualquier interpretación. Por un lado, si tratamos de mantenernos dentro de los límites de una epistemológica o metodología considerada mínimamente adecuada, el problema de cuáles son los entes teóricos a los que la mecánica cuántica se refiere parece no tener solución. Por otro lado, si empezamos por postular una ontología (o estructura lógica) mínimamente adecuada, extrayéndola directamente del formalismo, parece ser que no podemos relacionar tal nivel ontológico (o lógico) con una epistemología satisfactoria. Pero, en tanto no tengamos una interpretación adecuada de la mecánica cuántica, no podemos cimentar una interpretación de las probabilidades cuánticas en la estructura causal del mundo a nivel fundamental. Como veremos, esta conclusión se mantiene aun después de examinar las conclusiones de las pruebas de la no existencia de variables ocultas.

\section{Las pruebas de la imposibilidad de variables ocultas}

Variables ocultas establecen una relación entre magnitudes de dos teorías por medio de la cual podemos "reducir" una teoría a la otra. Los entes teóricos de la primera teoría se construyen, a partir de un teorema de representación, 
con base en los entes teóricos de la segunda teoría. La teoría a reducirse incluye magnitudes cuantitativas (variables) que son experimentalmente observables. La teoría reductora, por el contrario, incluye magnitudes (variables) no observables en la primera teoría. Si podemos describir a las magnitudes observables de la primera teoría (la teoría a reducirse) como "promedios" en algún sentido preciso (matemática y físicamente significativo) de los valores de las variables teóricas de la segunda teoría (la teoría reductora) entonces, se dice que, la teoría reductora es una teoría de variables ocultas para la primera. ${ }^{12}$ Es importante hacer notar que el "éxito" de una relación de variables ocultas como explicación de una relación entre teorías va a depender de los dos tipos de requerimientos interpretativos que introdujimos anteriormente. Por ejemplo, es relativamente fácil mostrar que existen teorías de variables ocultas que reproducen la distribución estadística de la mecánica cuántica por lo menos en ciertos casos simples. Lo que nadie ha podido encontrar es una teoría de variables ocultas que satisfaga ciertos requisitos mínimos generados por la interpretación física. ${ }^{13}$ Es claro entonces que para entender la idea de irreductibilidad tenemos que examinar con mucho cuidado el alcance y el significado de la relación por variables ocultas por medio de la cual podemos, supuestamente, efectuar una reducción del azar a una teoría determinista.

En su famoso tratado de 1932, en el cual se desarrolla la formulación que ahora es usual en el marco de la teoría de los espacios de Hilbert, Von Neumann presenta por primera vez un análisis matemático del proceso de

12 El paradigma de una relación por variables ocultas en la física es la reducción a la mecánica estadística clásica de la termodinámica. Sería tentador usar la terminología sugerida por este ejemplo y hablar de una teoría macroscópica (la teoría a reducirse) y de la teoría microscópica (la teoría reductora). Esta terminología sugeriría algo que no es cierto, esto es, que la relación por variables ocultas establece necesariamente una relación entre dos niveles epistemológicos que pueden reducirse a un nivel ontológico regido por leyes deterministas (véase Von Plato, 1982). No está de más mencionar que esta relación paradigmática por variables ocultas entre la mecánica estadística clásica y la termodinámica, aunque aparentemente bien establecida, es altamente controversial y ha sido el tema de discusiones filosóficas desde el momento que Boltzman la propuso originalmente. La idea de que tal relación es clara y firmemente establecida es uno de los mitos de la física y la filosofía de libros de texto.

13 Es famosa la propuesta de una teonía de variables ocultas de D. Bohm (1952). La mayoría de los físicos rechazan esta interpretación porque requiere la introducción de un potencial cuántico muy diferente de otros potenciales comunes en la fisica. En particular implica la existencia de efectos no locales (acción a distancia). Este potencial parece no tener otra motivación que el hecho que permite la formulación de una teoría de variabies ocultas. Además, la teoría de Bohm no parece compatible con una extensión relativista de la mecánica cuántica y en particular no parece constituir un marco conceptual viable para la electrodinámica cuántica, la aplicación más importante de la mecánica cuántica.

Existe también el problema, enfatizado por Muynck (1985), de que no es claro hasta qué punto la formulación de Bohr depende de la interpretación standard (subjetivista) del proceso de la medición. 
medición. Para asegurar la consistencia de su análisis con la supuesta universalidad de la teoría, se ve obligado a introducir un observador como componente indispensable del proceso de la medición.

Esta necesidad de introducir un elemento subjetivo en la descripción del proceso de medición llevaría naturalmente a una interpretación puramente subjetivista de la irreductibilidad de la teoría (véase Wigner, 1952, por ejemplo). Por lo menos en parte, este estado de cosas (que Von Neumann no consideraba satisfactorio) lo lleva a tratar de probar la imposibilidad de variables ocultas en una forma más directa, independiente del proceso de medición. Esta prueba es el contenido del famoso teorema de pariables ocultas de Von Neumann (1932). Aunque la formulación del teorema involucra una serie de resultados de la teoría de operadores en espacios de Hilbert la idea central del teorema es bastante simple. La intención de Von Neumann es mostrar que el algoritmo cuántico lejos de ser un simple postulado, es un principio que se sigue de consideraciones generales concernientes a la representación y relación entre magnitudes y estados estadísticos. El teorema ha sido desacreditado debido al hecho de que la prueba asume cierta estructura en el conjunto de magnitudes (por ejemplo, la aditividad de valores de esperanza matemática para operadores) que no puede motivarse sino recurriendo a la estadística cuántica (véanse Margenau y Park, 1973; Bell, 1966). El teorema de Von Neumann es importante de todas maneras porque trata de separar por primera vez los problemas con la interpretación de la teoría y en particular con el proceso de la medición, del problema más especifico de mostrar la no existencia de variables ocultas, y por consiguiente la de formular una version mínima del concepto de irreductibilidad a través de una prueba de la no existencia de variables ocultas.

\section{El enfoque lógico-algebraico}

Existen varias reformulaciones del resultado de Von Neumann basadas en hipótesis más débiles (y menos controversiales) que las asumidas por Von Neumann. La mayoría de ellas se originan en la reformulación de la mecánica cuántica en el marco de la teoría de retículos presentada en un trabajo conjunto de Von Neumann y Birkhoff (1936). Este enfoque establece una correspondencia entre enunciados descriptivos ("proposiciones") acerca de un sistema físico y los elementos de un retículo. A este retículo se le denomina, por analogía con la estructura proposicional clásica, "la lógica del sistema". Es posible demostrar que cada magnitud puede identificarse con un retículo de Boole y que un sistema cuántico tiene una lógica no booleana. La lógica de un sistema clásico constituye siempre un retículo booleano. Esto permite formular la diferencia entre la mecánica clásica y la mecánica cuántica sobre la base de su estructura lógico-algebraica. 
Recordemos que un retículo booleano puede siempre sumergirse (por medio de un mapeo homomórfico) en el retículo booleano 2 que consiste de dos elementos que pueden identificarse con los valores de verdad $T$ y $F$. Si podemos sumergir el retículo no booleano, que representa un sistema cuántico, en un retículo booleano, es decir, si podemos encontrar un homomorfismo que preserve la estructura lógica de las relaciones entre las magnitudes en consideración, entonces es posible redefinir las relaciones probabilistas de la teoría como derivadas de una medida clásica de probabilidad sobre un espacio (clásico) -aditivo. Kochen y Specker (1967) han mostrado que no existe tal homomorfismo y que, por lo tanto, el algortimo cuántico no puede reducirse a la teoría clásica de la probabilidad.

En otras palabras, en este enfoque la tesis de la irreductibilidad se identifica con la imposibilidad de mapear homomórficamente un reticulo no booleano en un reticulo booelano. La prueba de Kochen y Specker de que tal homomorfismo no existe es sin lugar a dudas un paso adelante, pero todavía se basa en cuestiones controversiales respecto a la interpretación de la teoría. Kochen y Specker, por ejemplo, asumen que es sólo sobre la base de una medición que podemos atribuir propiedades a sistemas. Puesto que esto no sucede (o por lo menos no parece suceder), a nivel macroscópico, su prueba de la no existencia de variables ocultas asume la partición del mundo en niveles, una tesis que hace eco de la idea de Bohr, pero que requiere una justificación que no se ofrece.

\section{El teorema de Bell}

J.S. Bell (véase Bell, 1966) probó un teorema que muestra la imposibilidad de variables ocultas sobre la base de una suposición bastante general y que puede formularse independientemente de aspectos problemáticos de la interpretación de la teoría, y en cierto sentido, incluso independiente de la teoría misma. El teorema de Bell se basa en un análisis de las correlaciones que aparecen en experimentos del tipo EPR. ${ }^{14}$ Bell impone la condición siguiente sobre toda teoría físicamente posible:

14 Una versión simple del experimento EPR es la siguiente. Supongamos que una partícula con valor cero de su magnitud spin (impulso angular intrínseco) decae en dos fotones $A$ y $B$. Las leyes de conservación implican que ambos fotones deben tener la misma polarización. El algoritmo cuántico predice que el fotón $A$ va a pasar un polarizador orientado en una dirección $d$ con una probabilidad $p$. Si suponemos que no hay acción a distancia es de esperarse que no exista correlación (o anticorrelación) entre las mediciones ejecutadas con polarizadores diferentes en las partículas $A$ y $B$ cuando éstas están suficientemente alejadas. Un polarizador podría estar en la ciudad de México y el otro en la luna, por ejemplo. Pero el hecho es que la mecánica cuántica predice tales correlaciones. En Rae (1986) hay una presentacion muy clara de este experimento y de su relación con el teorema de Bell. 
(i) Localidad (no acción a distancia). ${ }^{15}$ Las variables ocultas que determinan el valor de una magnitud para una de las dos partículas es independiente de la dirección del aparato de medición usado para la otra partícula.

(ii) La conservación de la energía.

(iii) Realismo (débil): las propiedades de sistemas físicos no dependen (por lo menos totalmente) del proceso de observación.

Estas condiciones son suficientes, como Bell demuestra, para generar distribuciones estadísticas incompatibles con las correlaciones que la mecánica cuántica predice (una prueba simple de este teorema se encuentra en Rae, 1986). El teorema de Bell tiene una importancia filosófica fundamental debido a que el argumento en favor de la irreductibilidad del algoritmo cuántico se puede establecer sobre una base que es independiente del proceso de la medición y, por lo tanto, independiente de los aspectos controversiales de la teoría, en particular, independiente de consideraciones epistémicas a la Bohr. Es más, el teorema de Bell no depende de ninguna hipótesis sobre la estructura lógica de los eventos en el mundo. Como Hellman (1982) nos dice, todo lo que es necesario aceptar son ciertos resultados experimentales que han sido ampliamente confirmados. Por un lado, expermentos que muestran correlaciones en polarización de fotones y, por el otro, la base experimental sobre la que se apoya la teoría especial de la relatividad, y en particular, la amplia base experimental para la existencia de un límite finito para la propagación de señales. Más precisamente, en tanto que una reducción por variables ocultas implica que para toda magnitud fisica, dado un valor inicial de esa magnitud probabilidades distintas de 0 y 1 son eliminables, el teorema de Bell muestra que la tesis reduccionista debe rechazarse (asumiendo, por supuesto, las hipótesis del teorema).

\section{Conclusion}

El teorema de Bell excluye definitivamente la posibilidad de construir teorías locales de variables ocultas para la mecánica cuántica y, en particular, nos dice que no podemos reducir las probabilidades del algoritmo cuántico en un mundo que es a la vez "realista" y "local". El teorema de Bell es un resultado profundo, pero como muchos otros resultados de tal generalidad (como el teorema de la incompletitud de Gödel en los fundamentos de la matemática) es un resultado de imposibilidad. El teorema de Bell no nos ayuda a entender las implicaciones ontológicas y epistemológicas de la ireducibilidad del algoritmo cuántico. Quizás, como sugiere Bell (en Davies y Brown, 1986),

15 Lo que esta condición en el teorema del Bell significa es el tema de una serie de discusiones actuales. Véase por ejemplo Redhead (1987). 
sea necesario pensar en una revisión de la teoría de la relatividad y volver a tomar seriamente la hipótesis del “éter”. Quizás debamos empezar a considerar seriamente la idea de la mente como un agente de la realidad física (como muchos físicos han sugerido). Ciertamente, y ésta es la conclusión de fondo para nuestro problema, si bien la irreductibilidad del algoritmo cuántico es un hecho en un mundo realista y local, esto no nos dice mucho respecto a las implicaciones filosóficas específicas de la irreductibilidad cuántica. En tanto que no tengamos una interpretación satisfactoria de la mecánica cuántica (u otra teoría más general) no parece que podamos decidir cuál es el importe epistemológico y cuál es el importe ontológico de la irreductibilidad del algoritmo cuántico.

Si bien, desde una cierta perspectiva histórico-metodológica, el desarrollo de la noción de "autonomía de leyes estadísticas" en la estructura de la ciencia culmina con la prueba de Von Neumann sobre variables ocultas (más correctamente, con el teorema de Bell), el problema filosófico que este desarrollo acarrea está lejos de ser comprendido en su totalidad. No podemos decir, por ejemplo, que conceptos cruciales como la explicación por medio de leyes estadísticas irreducibles sea una cuestión no problemática hasta que no clarifiquemos, en los casos paradigmáticos de tal tipo de leyes en la ciencia, en qué consiste esa irreductibilidad.

\section{BIBLIOGRAFIA}

Bell, J. S., 1966, "On the Problem of Hidden Variables in Quantum Mechanics", Review of Modem Physics, no. 38, pp. 447-452.

Bohm, D., 1952, “A Suggested Interpretation of the Quantum Theory in Terms of Hidden Variables", Physical Review, no. 85, pp. 166-180.

Bub, J., 1974, The Interpretation of Quantum Mechanics, Reidel, Dordrecht.

—_, 1977, "What is Philosophical Interesting about Quantum Mechanics?", en Procs.

of the fifth Int. Congr. of Logic, Methodology and Philosoplyy of Science, Springer Verlag, Berlín, vol. 10.

Davies, P.C., Brown, J.R., 1986, The Ghost in the Atom, Cambridge University Press, Cambridge.

DeWitt, B. y Graham, N. (comps.), 1973, The Many Worlds Interpretation of Quantum Mechanics, Princeton University Press.

Earman, J., 1986, A Primer of Determinism, Reidel, Dordrecht.

Einstein, A., Podolski y Rosen, 1935, Physical Revicw, no. 47.

Ekeland, I., 1984, Le calcul, Limprevu: Les figures du temps de Kepler a Thom, Eds. du Seuil.

Folse, H., 1985, The Philosophy of Niels Bolsr, Elsevier, 
Hacking I., 1983a, "The Autonomy of Statistical Law", en Scientific Explanation and Understanding, ed. N. Rescher, U. Press of America, Washington.

- 1983b, “Nineteenth Century Cracks in the Concept of Determinism”, Journal of the History of Ideas, vol. 44, no. 3.

- 1990, The Taming of Chance, Cambridge Univ. Press, Cambridge.

Healey, R., 1984, "How many Worlds?", Noiss, vol. 18, no. 4, pp. 591-616.

Hellman, G., 1980, "Quantum Logic and Meaning", Procs. of the Biennial Meeting of the Phil. of Science Assoc, vol. 2, pp. 493-511.

- 1982, "Einstein and Bell: Strengthening the case for microphysical randomness", Synthese, no. 53, pp. 445-460.

Jauch, J., 1968, Foundations of Quantum Mechanics, Addison Wesley, Reading Mass.

Keller, R., 1986, "The probability of Heads", Mathematical Monthly, marzo, pp. 191197.

Rae, A., 1986, Quantum Physics, Illusion or Reality?, Cambridge Univ. Press, Cambridge.

Readhead, M., 1987, Incompleteness, Nonlocality and Realism, Oxford Univ. Press, Oxford.

Von Neumann, J., Birkhoff, G., 1936, “The Logic of Quantum Mechanics”, Annals of Mathematics, no. 37.

Wigner, E., 1963, American J. of Physics, vol. 31, no. 6. 